\title{
InN Thin Film Lattice Dynamics by Grazing Incidence Inelastic X-Ray Scattering
}

\author{
J. Serrano, ${ }^{1, *}$ A. Bosak, ${ }^{2}$ M. Krisch, ${ }^{2}$ F. J. Manjón, ${ }^{3}$ A. H. Romero, ${ }^{4}$ N. Garro, ${ }^{5}$ X. Wang, ${ }^{6}$ A. Yoshikawa, ${ }^{7}$ and M. Kuball ${ }^{8}$ \\ ${ }^{1}$ ICREA-Departament de Física Aplicada, EPSC, Universitat Politècnica de Catalunya, \\ Carrer Esteve Terradas 5, E-08860 Castelldefels, Spain \\ ${ }^{2}$ European Synchrotron Radiation Facility, B.P. 220, 38043 Grenoble cedex 9, France \\ ${ }^{3}$ Instituto de Diseño para la Fabricación y Producción Automatizada, MALTA Consolider Team, \\ Universitat Politècnica de València, Camino de Vera s/n, 46022 València, Spain \\ ${ }^{4}$ CINVESTAV, Departamento de Materiales, Unidad Querétaro, Querétaro, Mexico 76230 \\ ${ }^{5}$ Materials Science Institute, University of Valencia, P.O. Box 22085, E46071 Valencia, Spain \\ ${ }^{6}$ School of Physics, Peking University, Beijing 100871, China \\ ${ }^{7}$ Graduate School of Electrical and Electronic Engineering, Chiba University 1-33 Yayoi-cho, Inage-ku, Chiba 263-8522, Japan \\ ${ }^{8}$ H. H. Wills Physics Laboratory, University of Bristol, Bristol BS8 1TL, United Kingdom
}

(Received 24 November 2010; published 19 May 2011)

\begin{abstract}
Achieving comprehensive information on thin film lattice dynamics so far has eluded well established spectroscopic techniques. We demonstrate here the novel application of grazing incidence inelastic x-ray scattering combined with $a b$ initio calculations to determine the complete elastic stiffness tensor, the acoustic and low-energy optic phonon dispersion relations of thin wurtzite indium nitride films. Indium nitride is an especially relevant example, due to the technological interest for optoelectronic and solar cell applications in combination with other group III nitrides.
\end{abstract}

DOI: 10.1103/PhysRevLett.106.205501

PACS numbers: 63.20.D-, 61.05.cf, 68.60.Bs

Indium nitride remains the least understood among the present technologically important semiconductors. The discovery of a $0.7 \mathrm{eV}$ direct band gap of this material $[1,2]$ and its combination with other group III nitrides is currently used for optoelectronic applications such as laser diodes and light-emitting diodes. The recent realization of $p$-type doping $[3,4]$ (in contrast to the commonly found $n$ type) in InN has opened new possibilities for the achievement of highly efficient solar cells. Despite the intense research carried out on $\mathrm{InN}$ electronic and vibrational properties, there is a lack of experimental information on its phonon dispersion relations [5]. Usually grown by molecular beam epitaxy (MBE), state-of-the-art high quality InN films rarely exceed $10 \mu \mathrm{m}$ thickness, thus preventing the use of standard bulk techniques to measure phonon dispersion relations. The available $\mathrm{InN}$ experimental data stem therefore mainly from Raman and infrared spectroscopy $[5,6]$, and new technical developments are required to complete this information over the entire Brillouin zone (BZ).

Whereas significant progress has been accomplished in the determination of lattice dynamics in single crystals, using both inelastic neutron (INS) and x-ray scattering (IXS) spectroscopies, there is a lack of corresponding techniques that can be employed for the study of thin films. Since phonons mediate the heat transfer processes in semiconductors, thin film lattice dynamics play a fundamental role in modeling thermal device behavior in the quest for more effective opto- and microelectronic semiconductor devices [7]. In this Letter, we demonstrate the potential of grazing incidence inelastic $\mathrm{x}$-ray scattering to obtain information on the lattice dynamics of micrometer thick thin films. We determine the complete elastic stiffness tensor and the acoustic and low-energy optic phonon dispersion relations of a $6.2 \mu \mathrm{m} \mathrm{InN} \mathrm{film} \mathrm{grown} \mathrm{on} \mathrm{GaN/sapphire.}$

The InN film was deposited at $600^{\circ} \mathrm{C}$ by molecular beam epitaxy on a (0001) sapphire substrate using a $0.5 \mu \mathrm{m} \mathrm{GaN}$ buffer layer. More details on the growth can be found in Ref. [8]. The film was unintentionally $n$ type doped, as common for InN, with a carrier concentration $n=4 \times 10^{18} \mathrm{~cm}^{-3}$ and a mobility $\mu=1420 \mathrm{~cm} / \mathrm{V} \mathrm{s}$. A rectangular shaped sample of $8 \times 5 \mathrm{~mm}$ surface was employed in the IXS experiment. The experimentally determined lattice parameters were $a=3.5340 \AA$ and $c=$ $5.7088 \AA$. These values are very close to the calculated ones (see below), therefore indicating that the $\mathrm{InN}$ film is fully relaxed. $\Omega$-scan x-ray diffraction on the same sample yielded rocking curves of 0.09 and 0.22 degrees full-widthat-half-maximum (FWHM) for the 002 and 102 reflections, respectively. These widths are significantly larger than expected from the intrinsic reflection width and the $\mathrm{x}$-ray beam divergence, and are most likely due to the natural mosaicity of the sample. Figure 1(a) displays a scanning electron microscopy (SEM) image of the film section, showing the $\mathrm{InN}$ layer on top of the $\mathrm{GaN}$ buffer layer and the sapphire substrate. Indeed, the image clearly reveals the internal structure of the InN film with individual mosaic blocks and various defects.

Grazing incidence room temperature IXS experiments were performed on beam line ID28 at the European Synchrotron Radiation Facility, using an incident photon energy of $17.794 \mathrm{keV}$, as obtained from a Si 999 monochromator working in nearly backscattering geometry, and achieving $3 \mathrm{meV}$ FWHM energy resolution. The beam was 


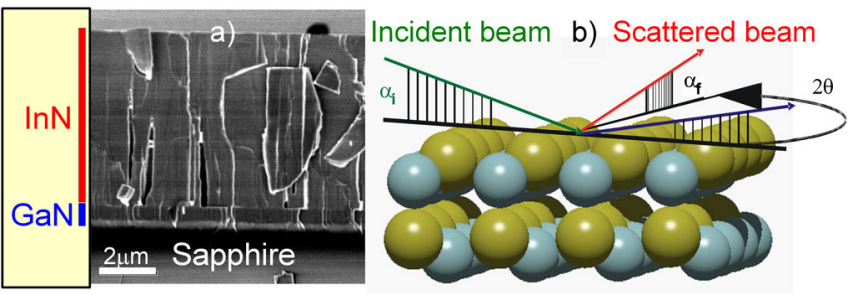

FIG. 1 (color online). (a) SEM image of the wurtzite InN film. (b) Grazing incidence IXS geometry, where $\alpha_{i}$ and $\alpha_{f}$ are $1.7^{\circ}$. The $2 \theta$ angle determines the total momentum transfer selected by the spectrometer.

focused to a $60 \times 250 \mu \mathrm{m}^{2}$ beam size (vertical $\times$ horizontal, FWHM) employing a toroidal mirror. A flat platinum-coated mirror was mounted in front of the sample in order to deflect the x-ray beam downwards. The glancing angle of incidence, $\alpha_{i}$, was adjusted to $1.7^{\circ}$ [see Fig. 1(b)]. In this geometry the observed scattering intensity arises predominantly from the InN film (99.9\%). The constraints of the grazing incidence geometry and the fact that the film normal is parallel to the $c$ axis implies that only longitudinal and transverse in-plane $(a-b)$ phonon modes can be recorded. The phonon dispersion along $\Gamma-A$ and a few IXS scans for the determination of the elastic constant $C_{13}$ were recorded in a configuration with a larger glancing angle of incidence. Taking into account the ratio of the film thickness, the absorption through the $\mathrm{InN}$ film, and the scattering signal from $\mathrm{InN}$ and $\mathrm{GaN}$, the maximum contribution of the GaN film to the IXS signal is at most $1.3 \%$. For all the employed configurations, the contribution of the GaN buffer and the sapphire substrate can therefore be safely neglected.

$A b$ initio calculations of $\mathrm{InN}$ phonon dispersion relations and phonon eigenvectors were performed to identify the Brillouin zones suitable to achieve highest IXS intensity, according to the selection rules, and to analyze the experimental data. Calculations were based on density functional theory as implemented within the ABINIT software package [9-11]. Both local density approximation (LDA) and generalized gradient approximations (GGA) were employed to ascertain the changes in the elastic stiffness tensor and the phonon dispersion relations with the exchange and correlation energy. Only the valence electrons were taken into account by using Troullier-Martins [12] and HartwigsenGoedecker-Hutter pseudopotentials [13] for LDA and GGA, respectively. A $60 \mathrm{Ha}$ plane-wave energy cutoff was used within both LDA and GGA, combined with a $6 \times 6 \times 3 k$-point mesh for integration in order to ensure convergence of both total energy and stress tensor. The $k$-point grid was required to be increased further up to $14 \times 14 \times 8$ to improve the convergence for the elastic stiffness tensor. The ground state geometry was optimized relaxing both internal parameter and lattice constants at constant pressure up to a value of $3 \times 10^{-2} \mathrm{GPa}$. The phonon frequencies and eigenvectors were calculated

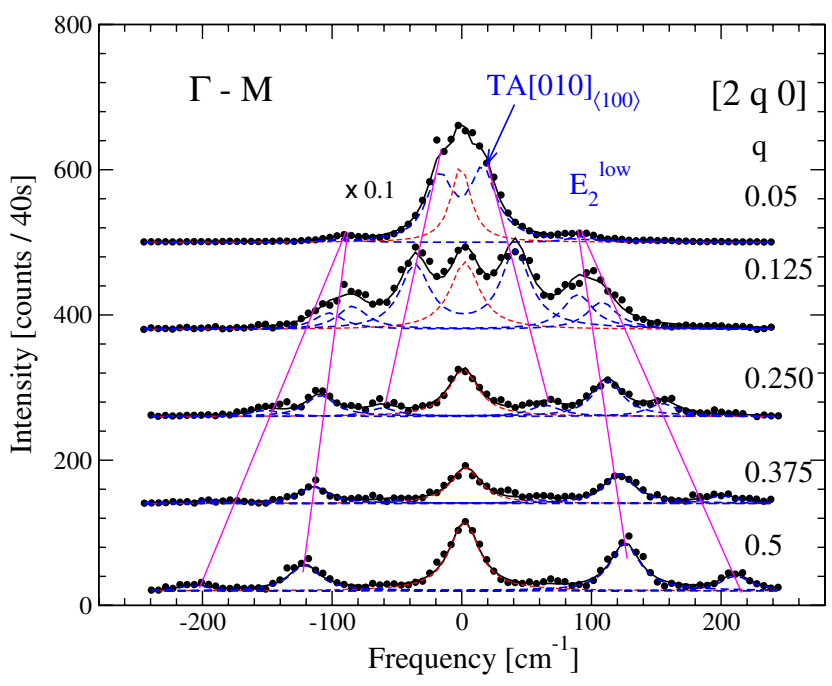

FIG. 2 (color online). Selected IXS spectra for momentum transfer values along the $\Gamma-M$ direction of the BZ. The symbols display the experimental data whereas the solid curves represent the best fit to the spectra. Dashed lines are used to indicate the contributions of different excitations and the zero-energy central elastic peak. The solid straight lines are guides to the eye to highlight the different phonon branch dispersions with increasing momentum transfer. The $\xi=0.05$ spectrum intensity has been multiplied by a factor 0.1 to better display the mode dispersion. Note the drastic decrease in TA intensity with increasing momentum transfer.

using Fourier interpolation of a set of dynamical matrices calculated via nonlinear response on a $6 \times 6 \times 3$ grid of $q$ points in the irreducible BZ.

Figure 2 shows a representative set of selected IXS spectra of transverse phonons along the [010] direction of the BZ, i.e., $\Gamma-M$ direction. The IXS signal is plotted as a function of momentum transfer $q$, with respect to the 200 Bragg peak. Besides the central elastic peak, a number of maxima appear at positive and negative energy transfers corresponding to phonon creation and annihilation processes, respectively. The transverse acoustic modes (TA) can be clearly distinguished from two higher energy dispersing excitations that stem from the $E_{2}^{\text {low }}$ mode at $q=0$. In this case, the $E_{2}^{\text {low }}$ intensity is more strongly dependent on the change of phonon eigenvectors with momentum transfer. A Lorentzian function of $3 \mathrm{meV}$ FWHM was used to fit the elastic peak, while pairs of Lorentzians with adjustable FWHM were utilized to obtain the frequency of the phonon excitations.

Similar to Brillouin scattering, inelastic x-ray scattering allows one to probe the momentum transfer dependence of acoustic branches and hence derive the corresponding elastic stiffness constants. The advantage of using grazing incident IXS lies in the feasibility to obtain accurate information in thin films deposited on a substrate for nearly all directions of propagation, without the need to detach the film from the substrate. Grazing incidence IXS 
spectroscopy, in contrast to other techniques, is capable of selecting the probing depth in the film by varying the glancing angle. It therefore provides a unique way to investigate substrate-induced strain effects on the elastic constants even in submicron thick films.

In order to obtain the elastic constants, we first fitted the acoustic phonon branches at low $q$ with a sinusoidal function $\omega(q)=A \sin (q \pi / B)$, where $A$ and $B$ are the fitting parameters. The limit for $q=0$ of the slope of such function provides the sound velocity for the corresponding phonon branch. The sound velocity $V$ is related to either a single or a combination of components of the elastic stiffness tensor via the Christoffels equation [14]. There are 5 independent components in the elastic stiffness tensor for the wurtzite structure: $C_{11}=\rho V(L A[100])^{2}$, $C_{33}=\rho V(L A[001])^{2}, C_{66}=\rho V\left(T A[010]_{(2-10\rangle}\right)^{2}, C_{44}=$ $\rho V\left(T A[100]_{(001\rangle}\right)^{2}$, and $C_{13}$, where $\rho$ is the mass density. Taking the experimental values for the lattice constants found in the IXS experiment we employed a mass density $\rho=6.93 \mathrm{~g} / \mathrm{cm}^{3}$ for the derivation of the elastic constants. $C_{13}$ can only be obtained as a component in the sound velocity along a direction neither in-plane nor aligned with the $c$ axis, and it was obtained by measuring the phonon energy of LA modes at small momentum transfer along the $\left[\begin{array}{lll}2 & 0 & 3\end{array}\right]$ direction and solving the Christoffel equation selfconsistently.

Table I lists the elastic stiffness tensor obtained from the IXS data and compares them with results from our calculations and previously reported experimental [15] and $a b$ initio calculated data [16-19]. Note the large discrepancy between the values determined by IXS and those reported in Ref. [15] from temperature-dependent $x$-ray diffraction broadening in polycrystalline InN. Our experimental values are in very good agreement with the calculations reported in Refs. [17-19] and our own

TABLE I. Elastic stiffness tensor obtained by IXS, other experimental data, and ab initio calculations, in GPa. For completeness, we include the values for the bulk modulus $B$ and the biaxial elastic modulus $G_{a b}=C_{11}+C_{12}-2 C_{13}^{2} / C_{33}$.

\begin{tabular}{|c|c|c|}
\hline IXS & Other experiment & Other calculations \\
\hline & & LDAGGA \\
\hline$C_{11} 225(7)$ & $190(7)^{\mathrm{a}}$ & $224238271^{\mathrm{b}}, 232^{\mathrm{c}}, 223^{\mathrm{d}}, 229^{\mathrm{e}}$ \\
\hline$C_{12} 109(8)$ & $104(3)^{\mathrm{a}}$ & $112112124^{\mathrm{b}}, 115^{\mathrm{c}}, 115^{\mathrm{d}}, 120^{\mathrm{e}}$ \\
\hline$C_{13} 108(8)$ & $121(7)^{\mathrm{a}}$ & $94^{\mathrm{b}}, 96^{\mathrm{c}}, 92^{\mathrm{d}}, 95^{\mathrm{e}}$ \\
\hline$C_{33} 265(3)$ & $182(6)^{\mathrm{a}}$ & $243237200^{\mathrm{b}}, 239^{\mathrm{c}}, 224^{\mathrm{d}}, 234^{\mathrm{e}}$ \\
\hline$C_{44} \quad 55(3)$ & $10(1)^{\mathrm{a}}$ & $46^{\mathrm{b}}, 52^{\mathrm{c}}, 48^{\mathrm{d}}, 49^{\mathrm{e}}$ \\
\hline$C_{66} \quad 58(2)$ & $43(5)^{\mathrm{a}}$ & $74^{\mathrm{b}}, 59^{\mathrm{c}}, 54^{\mathrm{d}}, 55^{\mathrm{e}}$ \\
\hline B $152(5)$ & $139^{a}$ & $142145147^{\mathrm{b}}, 151^{\mathrm{c}}, 141^{\mathrm{d}}, 146^{\mathrm{e}}$ \\
\hline$G_{a b} 245(13)$ & $133^{\mathrm{a}}$ & $268279307^{\mathrm{b}}, 270^{\mathrm{c}}, 262^{\mathrm{d}}, 271^{\mathrm{e}}$ \\
\hline
\end{tabular}

${ }^{a}$ Temperature-dependent x-ray diffraction broadening, Ref. [15]. ${ }^{b}$ LDA, full-potential linear-muffin-tin-orbitals (FP-LMTO) and tensor transformations, Ref. [16].

${ }^{\mathrm{c}}$ LDA, FP-LMTO, Ref. [17].

${ }^{\mathrm{d}}$ LDA, plane-wave pseudopotentials, Ref. [18].

${ }^{\mathrm{e}}$ LDA, plane-wave pseudopotentials, Ref. [19]. calculations. The use of the LDA exchange and correlation term results in a significant improvement of the calculation of the axial elastic modulus, $C_{33}$, which stems from the longitudinal sound velocity propagating along the $c$ axis. The calculated value for $C_{33}$ is particularly affected by the $k$-point grid employed, decreasing in value with increasing number of $k$ points. Our convergence tests ensure reliable results with a margin of $3 \mathrm{GPa}$ for this elastic constant.

Figure 3 displays the phonon dispersion relations determined in $\mathrm{InN}$ up to $250 \mathrm{~cm}^{-1}$, which are dominated by atomic displacements of the indium sublattice. Different symbols are used to plot IXS data originating from different scattering polarization geometries. Both GGA and LDA approximations yield phonon frequencies (solid and dashed curves) in excellent agreement with the experimental data (symbols). Our LDA results are essentially identical to earlier calculations by Bungaro et al. [20]. We were able to determine the value at the $\Gamma$ point, $230(1) \mathrm{cm}^{-1}$, and the dispersion of the lower energy silent $B_{1}$ mode, which appears frequently in Raman spectra of doped nitrides due to the induced lattice disorder [21]. Most of the data (purple or light gray solid circles) were taken using an in-plane scattering geometry with mixed longitudinal and transverse character, taking full advantage of the multianalyzer spectrometer of ID28. The (red) squares display data

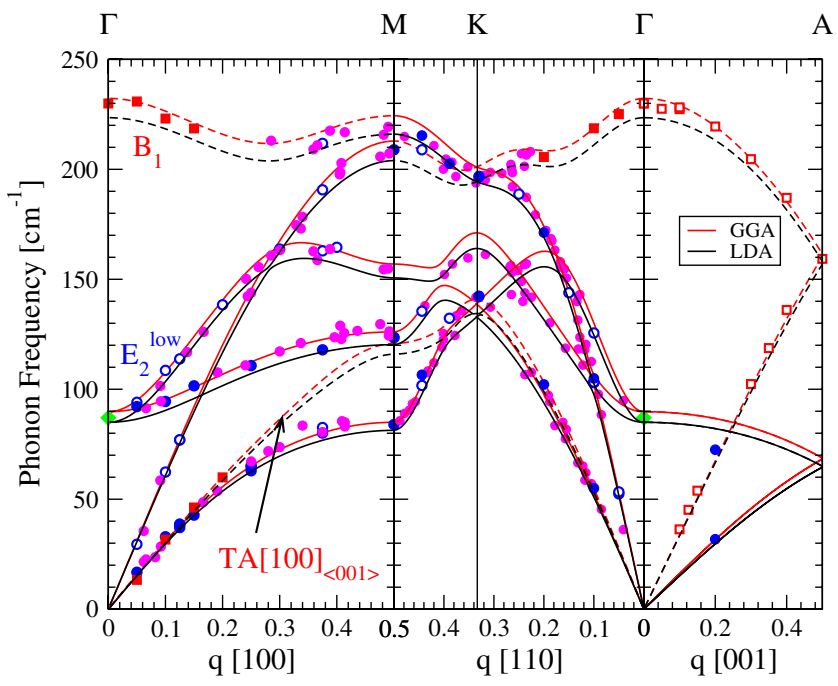

FIG. 3 (color online). Acoustic and low-energy optic phonon dispersion relations of wurtzite $\mathrm{InN}$ along the main symmetry directions. Open and solid symbols display IXS data corresponding to longitudinal and transverse modes, respectively, except for the solid circles that display IXS data obtained using mixed transverse and longitudinal polarizations. The black (red) curves plot results obtained from LDA (GGA) ab initio calculations. Modes polarized along the $\langle 001\rangle$ direction of the BZ are represented by (red) squares and dashed lines, whereas in-plane polarized modes are represented by (blue) circles and solid lines. The green diamond at the $\Gamma$ point shows Raman data for the $E_{2}^{\text {low }}$ mode from Ref [6]. Momentum transfers are given in reciprocal lattice units. 
obtained with an IXS scattering geometry involving modes polarized along the $c$ axis, whereas the dashed curves display the calculated phonon branches with eigenvectors at the $\Gamma$ point polarized along the same axis. There is excellent agreement between experimental and calculated data. Raman data for the lower energy $E_{2}$ mode, $87 \mathrm{~cm}^{-1}$, reported by Davydov and co-workers [6] are also in agreement with the extrapolation of IXS data at low momentum transfers, 90(1) $\mathrm{cm}^{-1}$.

In conclusion, this work demonstrates the feasibility of grazing incidence IXS to provide valuable information on the lattice dynamics of thin films, with an interest in many fields of material science, such as novel advanced materials, semiconductors, and superconductors. We have discussed results on the phonon dispersion relations and the elastic stiffness tensor of a thin film of InN. The five independent components of the elastic stiffness tensor have been obtained in a self-consistent manner and compared with calculations performed using first-principles methods. Phonon frequencies and polarizations have been determined for the six lowest energy branches of the InN phonon dispersion relations, providing valuable information about the energy and dispersion of the silent $B_{1}$ mode at $230 \mathrm{~cm}^{-1}$. The experimental data have been analyzed with the aid of $a b$ initio calculations. The calculated phonon frequencies, polarizations, and elastic constants display an excellent agreement with the experimental data. We hope that this work will stimulate further experimental studies of phonon dispersion relations and elastic constants in thin films.

J.S. acknowledges financial support from the Spanish Ministry of Science and Innovation by CICYT Grants No. MAT2010-2-129-C02-01 and No. ENE2008-04373, and by Generalitat de Catalunya Grant No. 2009SGR1251. F. J. M. is thankful for the financial support from CICYT projects No. CSD2007-00045 and No. MAT2010-21270-C04-04, and the "Programa de Incentivo a la Investigación" of the Universidad Politécnica de Valencia through project No. UPV20100096. A. H. R. has been supported by CONACyT Mexico under projects No. TAMU-Conacyt and No. J-83247-F. We acknowledge the beam time granted by ESRF. *jserrano@fa.upc.edu

[1] J. Wu, W. Walukiewicz, K. M. Yu, J. W. Ager III, E. E. Haller, H. Lu, W. J. Schaff, Y. Saito, and Y. Nanishi, Appl. Phys. Lett. 80, 3967 (2002).

[2] V. Y. Davydov et al., Phys. Status Solidi (b) 229, R1 (2002).

[3] R.E. Jones, K. M. Yu, S.X. Li, W. Walukiewicz, J. W. Ager, E. E. Haller, H. Lu, and W. J. Schaff, Phys. Rev. Lett. 96, 125505 (2006).

[4] J.-H. Song, T. Akiyama, and A. J. Freeman, Phys. Rev. Lett. 101, 186801 (2008).

[5] Z. Qian, W. Shen, H. Ogawa, and Q. Guo, J. Phys. Condens. Matter 16, R381 (2004).

[6] V. Y. Davydov, V. V. Emtsev, N. Goncharuk, A. N. Smirnov, V. D. Petrikov, V. V. Mamutin, V. A. Vekshin, and S. V. Ivanov, Appl. Phys. Lett. 75, 3297 (1999).

[7] A. Majumdar, J. Heat Transfer 115, 7 (1993).

[8] K. Xu and A. Yoshikawa, Appl. Phys. Lett. 83, 251 (2003).

[9] X. Gonze et al., Comput. Mater. Sci. 25, 478 (2002).

[10] X. Gonze et al., Z. Kristallogr. 220, 558 (2005).

[11] X. Gonze et al., Comput. Phys. Commun. 180, 2582 (2009).

[12] N. Troullier and J.L. Martins, Phys. Rev. B 43, 1993 (1991).

[13] C. Hartwigsen, S. Goedecker, and J. Hutter, Phys. Rev. B 58, 3641 (1998).

[14] B. A. Auld, Acoustic Fields and Waves in Solids (Krieger Publishing, Malabar, 1990), Vols. I and II, 2nd ed.

[15] A U. Sheleg and V. A. Savastenko, Izv. Akad. Nauk SSSR, Neorg. Mater. 15, 1598 (1979) [Inorg. Mater. (USSR) 15, 1257 (1979)].

[16] K. Kim, W. R. L. Lambrecht, and B. Segall, Phys. Rev. B 53, 16310 (1996).

[17] K. Sarasamak, S. Limpijumnong, and W. R. L. Lambrecht, Phys. Rev. B 82, 035201 (2010).

[18] A. Wright, J. Appl. Phys. 82, 2833 (1997).

[19] S. P. Lepkowski, J. A. Majewski, and G. Jurczak, Phys. Rev. B 72, 245201 (2005).

[20] C. Bungaro, K. Rapcewicz, and J. Bernholc, Phys. Rev. B 61, 6720 (2000).

[21] F. J. Manjón, B. Marí, J. Serrano, and A. H. Romero, J. Appl. Phys. 97, 053516 (2005). 